\title{
Sistemas responsivos: relevância, estado da arte e desenvolvimentos
}

\author{
Responsive systems, relevance, state of the art and developments
}

\author{
- Gonçalo Castro Henriques \\ Universidade Federal Rio de Janeiro, Brasil \\ gch@ufrj.br
}

\begin{abstract}
Responsive architecture is often seen as one that merely adapts to change. This reflects its limited and still incipient application in architecture. Given the current resource's crisis, a systemic building management is essential. This article argues that there is no established process for creating and managing responsive architecture. Therefore, it claims is necessary to deepen knowledge about systems, computation, mathematics, biology and robotics. Despite being a vast subject, it proposes a 'state of the art' about systems, investigating how to operate them. Based on this, proposes a method for generating responsive systems. This method is tested in a practical case.
\end{abstract}

Keywords: Responsive Systems, Meta-Systems, Static Adaptation, Dynamic Adaptation, Heuristics

\section{Introdução: problema, relevância e objetivos}

As tecnologias digitais permitem utilizar melhor os recursos, que são escassos e finitos. Em arquitetura a sua utilização permite integrar diferentes processos, encurtando o ciclo produtivo, ganhando eficiência. As tecnologias digitais, para além de permitem gerir o processo de conceção e fabricação, permitem também gerir os edifícios depois de construídos. No entanto não só há pouco edifícios que tenham essa gestão, como a gestão em rede não existe. A atual crise energética e de recursos tornam fundamental reduzir os gastos energéticos. Os edifícios gastam energia significativa quer durante a construção, quer na sua manutenção. Tal torna prioritário conceber sistemas de gestão de energia. O conhecimento sobre sistemas beneficiou do avanço da teoria geral dos sistemas e do desenvolvimento da teoria de controlo dos sistemas, a cibernética. No entanto estes temas têm sido pouco explorados em arquitetura. Argumenta-se que tal se deve a serem necessários conhecimentos de áreas como matemática, computação, biologia e robótica. Esta pesquisa pretende identificar os saberes multidisciplinares necessários para definir sistemas responsivos. Para tal tratará de identificar, analisar e sintetizar os fundamentos necessários para a sua implementação. Com base nessa informação propõe um método para obter um sistema responsivo, que será testado na prática.

\section{Metodologia teórico-prática}

O artigo começará por referir a importância da redução energética e da necessidade de uma arquitetura responsiva. Abordará depois alguns avanços em diferentes áreas de conhecimento capazes de informar a ideia concetual e operativa de sistema. Com base nesta pesquisa irá propor um método para desenvolver sistemas responsivos, testando-os em seguida, através de uma aplicação prática.

A sociedade atual enfrenta sérios desafios ambientais. Edward Mazria estudou a relação entre arquitetura, energia e meio ambiente. Mazria contabilizou nos EUA o consumo de energia em três setores básicos: arquitetura, transportes e

indústria. Segundo este estudo, o setor de arquitetura consome $48 \%$ da energia, o de transportes $27 \%$ e a indústria $25 \%$. Sendo que, por outro lado, o setor da arquitetura gera $46 \%$ das emissões anuais $\mathrm{CO} 2$, número significativo e que tende a aumentar. Esta tendência pode alargar-se às cidades em geral, o que justifica uma reflexão sobre este impacto. Explica também a preocupação crescente com a redução do consumo de energia na arquitetura e construção. Neste contexto, e para um ambiente mais sustentável são necessários edifícios que se adaptem ao meio e utilizem cada vez menos recursos. E os edifícios podem adaptar-se melhor ao meio ambiente através dos processos integrados digitais.

Desenvolvimentos na biologia, na computação e 
arquitetura permitem pensar edifícios responsivos e que se adaptam dinamicamente ao meio, ao contrário dos edifícios tradicionais e muito estáticos. Tal implica uma visão ecológica de populações, com enfase nas regras e na interação do conjunto. Exige ultrapassar uma visão parcial, centrada em cada objeto como entidade isolada - antes passando a considerar os edifícios em rede. São, assim, necessárias novas ferramentas que organizem e regulem o fluxo de informação e que permitam que os edifícios se ajustem ao meio de acordo com as variações climáticas, geográficas, horárias, entre outras. Esta capacidade de adaptação do conjunto e individualmente permitirá reduzir o consumo energético.

\section{Sistema responsivo, estado arte}

Após a revolução industrial houve uma especialização do saber técnico e científico. Tal evidenciou a necessidade de uma teoria que considerasse o conhecimento holísticamente. Assim surgiu a teoria geral dos sistemas que considera holísticamente diversos ramos do conhecimento, como a biologia, sociologia ou a computação. Partindo desta teoria contribuíram para o desenvolvimento da arquitetura responsiva diversos protagonistas de diferentes áreas como Bertalanffy, Pask, Alexander, Weinstock, Negroponte, Sterk, DeLanda e Achten entre outros.

A «teoria geral dos sistemas» desenvolvida por Von Bertalanffy (1950-68) é aplicável a sistemas vivos, sociais ou máquinas. Bertalanffy definiu matematicamente os mecanismos de adequação dos sistemas, como o feedback e a equifinalidade. Gordon Pask desenvolveu a teoria de controlo dos sistemas (cibernética, 1969), completando os mecanismos
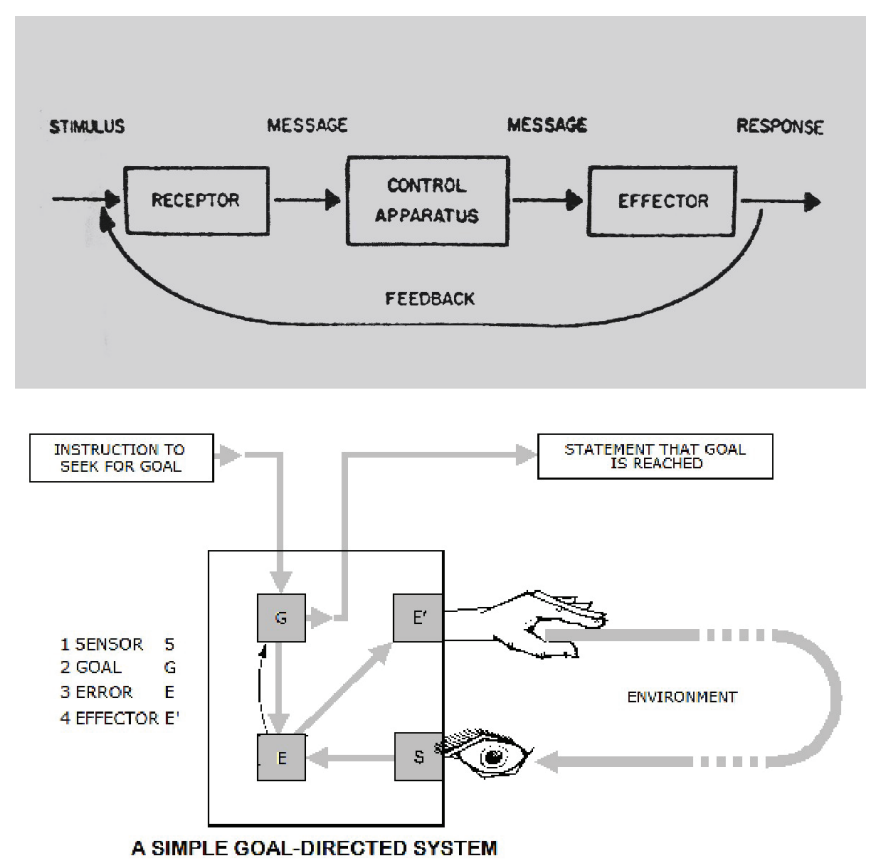

Figura 1: Mecanismo simples de feedback (Ludwig Von Bertalanffy, 1969) e Mecanismo Feedback orientado por um objetivo (Gordon Pask, 1969). de controlo de Bertalanffy com a "procura por objetivos". Se as máquinas eram encaradas como sistemas fechados, com o desenvolvimento da cibernética, passaram a poder ser consideradas como sistemas abertos, desde que sejam capazes de apreender e de se adaptarem ao meio tal como o fazem outros sistemas vivos. Estas definições permitiram passar de sistemas fechados e unidirecionais para sistemas abertos multicausais. A mencionada teoria suporta a criação de ambientes colaborativos entre o homem e a máquina, fundamentais para uma arquitetura responsiva.

Christopher Alexander reflete sobre a relevância da palavra 'sistema' em arquitetura, no seu texto "sistemas gerando sistemas" (1968). Reflete também como criar sistemas holísticos que garantam formas sincronizadas com o seu ambiente. Alexander afirma que um sistema só pode ser considerado como tal, se possuir um sistema generativo que regule a relação das partes com o todo e do todo com as partes. Destaca que os sistemas generativos são essenciais para definir sistemas holísticos.

Nicholas Negroponte é um dos pioneiros da arquitetura responsiva nos anos 70. Antevê como as tecnologias digitais podem melhorar os sistemas de documentação, os sistemas generativos de projeto e como podem suportar espaços inteligentes, incorporando o computador. No entanto segundo Tristan-d'Estree Sterk esse entendimento teórico atualmente nãoé suficientepara implementar uma arquitetura responsiva. Segundo ele "A arquitetura responsiva é frequentemente definida como um tipo de arquitetura que tem a capacidade de alterar a sua forma, em resposta às novas condições. Embora esta descrição capture com sucesso a essência do tema, não fornece um entendimento detalhado necessário para a construir." Argumenta que para tal é fundamental associar conhecimentos de arquitetura, de inteligência artificial, robótica e engenharia estrutural. A ausência de saberes nestas áreas pela maioria dos arquitetos justifica que apresentem uma arquitetura responsiva ambivalente, tendo ainda dificuldade em ultrapassar uma estética que seja mais do que algo baseado em eventos.

Sterk defende também que é necessário entender a arquitetura responsiva em diferentes escalas. Refere como o modelo precedente, de Negroponte, é interessante concetualmente, mas que ainda escasseiam modelos computacionais. Destaca os avanços nos campos da inteligência artificial e robótica, com a definição de sistemas de controlo de alto-nível (deliberativos) e de baixo-nível (auto-regulados). Propõe implementar uma arquitetura responsiva com base num sistema de controlo híbrido (Fig. 2) combinando computação de alto-nível (deliberativa) com computação de baixo-nível (auto-regulada). No entanto, pode argumentar-se que Pask - ao propor uma aproximação por objetivos - já estabelecera ciclos de baixo-nível dentro de ciclos deliberativos de alto-nível (Fig.1). Este encadeamento permite associar a capacidade deliberativa com a autorregulação. As respostas de baixo nível podem ser dadas através de sensores e quando um determinado valor é atingido, é então executada 

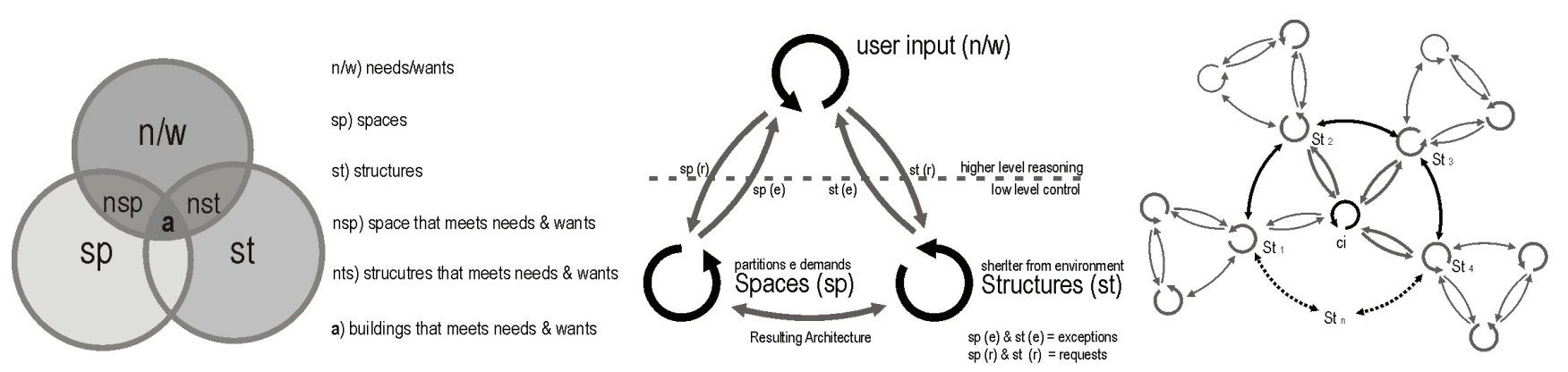

Responsive Architecture Hybridized Model Control Sterk: 1 The three components of a discrete model of architecture. 2 The proposed hybridized control model for use within a functional responsive architecture. 3 The framework of a responsive network that stretches across a cluster of buildings.

Figura 2: Modelo de controlo híbrido para uma arquitetura responsiva, Sterk (2006). Combina processos de alto nível (deliberativos) e de baixo nível (auto-regulados). Da esquerda para a direita: 1 Componentes de um modelo discreto de arquitetura que incluem os desejos e necessidades $(\mathrm{n} / \mathrm{w})$, com espaços (sp) e estruturas (st); 2 Decisões de alto nível usuário (n/w), espaço (sp) estrutura (st) sendo que dentro de cada uma há decisões de baixo-nível; 3 arquitetura responsiva aplicada numa rede de edifícios, repetindo a combinação anterior mas numa escala maior.

uma ordem de alto nível. Neste processo encadeado o mecanismo é essencial o mecanismo de feedback.

Pretende combinar o controlo deliberativo, citando o proposto por Yona Friedman (1971), com o controlo autoregulado tal como proposto por Chuck Eastman (1972). Enquanto o controlo deliberativo exige computação de alto nível (ou seja a utilização de uma linguagem codificada e que o usuário conheça o funcionamento do sistema), o controlo auto-regulado não exige a utilização de uma linguagem codificada podendo ser controlado por linguagem de máquina. No controlo auto-regulado são utilizados procedimentos reflexivos simples, sendo o estado do sistema resultante da interação entre as partes. Mike Weinstock destaca no fenómeno da emergência a importância da definição matemática. Apresenta uma teoria sobre a morfogénese da forma designada como Teoria da Emergência (2004).

Henry Achten identificou várias definições relacionadas com a arquitetura responsiva, nomeadamente: sistema de automação de edifício, smart home, edifício senciente, edifício adaptativo, edifício dinâmico, edifício interativo, arquitetura cinética, edifício inteligente e edifício portátil. Achten apresenta uma definição da aplicação destes termos, concluindo que não existir uma classificação unanime porque cada um dos termos está relacionado com uma aplicação tenológica específica. Refere que devido a estas tecnologias serem recentes ainda não foram absorvidas pela linguagem comum. Achten carateriza também a interação dos sistemas responsivos com o usuário, defendendo o recurso a desenvolvimentos recentes da inteligência artificial. Nomeadamente a utilização de redes multiagentes, capazes de suportar a análise, o projeto e o desenvolvimento de sistemas complexos em todas as fases de projeto. Reforça assim a necessidade referida por Sterk, de que arquiteto necessita de conhecimentos mais vastos para este tipo de arquitetura.

\section{Como gerar sistemas responsivos}

Para suportar uma arquitetura responsiva é necessário criar sistemas que gerem sistemas, ou seja metasistemas com as características que se pretende introduzir nesses sistemas responsivos. Pask, na teoria dacibernética, refereser necessário que os arquitetos gerem sistemas que gerem edifícios, em vez de simplesmente desenharem edifícios. Alexander, analisando sistemas em arquitetura, também concorda - dizendo que para gerar sistemas há que conceber os sistemas generativos que os capacitam. Autores de diferentes áreas de conhecimento coincidem que são necessários metasistemas com sistemas generativos. Sterk chama a atenção que para controlar estes metasistemas são necessários conhecimentos de diferentes áreas. No entanto apesar de Sterk propor um modelo ainda não é claro como poderá ser a sua implementação.

DeLanda refere que um sistema tem capacidades que advêm da interação das partes e que superam as suas propriedades individuais. Refere que para estas capacidades emergirem é necessário definir os procedimentos e as variáveis do sistema. Refere que são as restrições aplicadas às variáveis que definem progressivamente os sistemas. Considera que os "algoritmos são a essência do software", referindo nesse sentido que a matemática metaforicamente é um tipo de software. Cita a evolução da física na definição de sistemas, nomeadamente a definição da gravidade proposta por Richard Feynman em três momentos: 1 força e aceleração, 2 campos de forças, 3 singularidades e espaço de soluções (mínimo, máximo, características variacionais). Esta mudança está relacionada com a matemática das quantidades para a matemática qualitativa, de valores para parâmetros. Esta mudança também está relacionada com a passagem da geometria euclidiana e dos sistemas métricos, para a geometria topológica dos espaços locais. Esta matemática permite definir comportamentos associativos entre as partes através de parâmetros, que passam a poderem ser codificados através de algoritmos. 
Os exemplos de sistemas naturais e da biologia têm vindo a ser incorporados na computação para definir sistemas abertos, que estão na base dos sistemas responsivos. Os sistemas abertos têm a capacidade de se retro alimentarem através de mecanismos de feedback e de se adequarem, reconfigurando a relação entre as suas partes. Os sistemas vivos possuem uma hierarquia com diferentes níveis de definição que permitem controlar sistemas complexos, como por exemplo corpo humano, sistema ósseo, sistema muscular, tecidos, células. A relação entre sistemas é hierárquica mas também emergente, do todo para a parte e da parte para o todo.

\section{Método arquitetura responsiva}

Este estudo aprofundou o estado da arte da arquitetura responsiva, relacionando contributos dispersos procurando informação de como fundamentar e gerar um 'sistema responsivo'. Na sequência desse trabalho, é proposto um método para gerar sistemas responsivos, que será testado através de um caso prático.

\subsection{Metasistema}

Para gerar um sistema responsivo é proposto um metasistema, que orienta o campo de pesquisa, sendo necessário para formalizar uma solução. Para que o método fique mais claro será aplicado num caso prático.

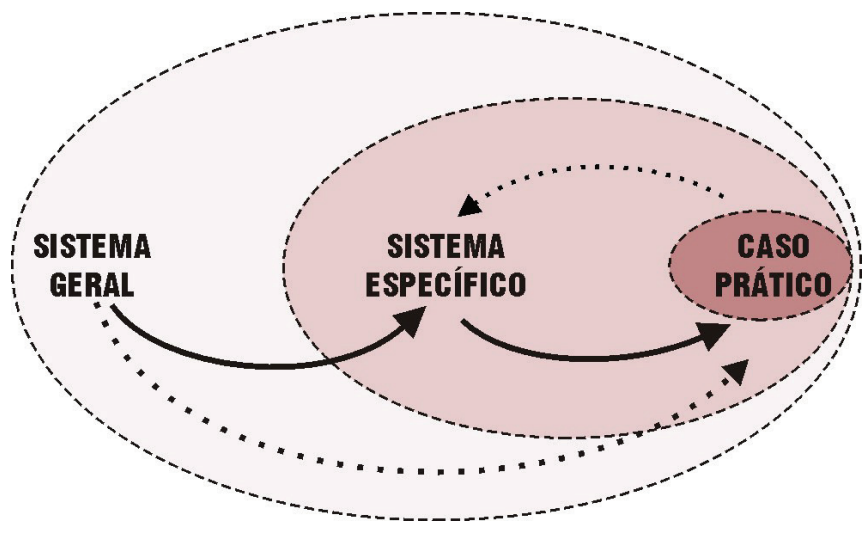

Figura 3: Metasistema proposta para formalizar um sistema responsivo, definindo o espaço de pesquisa do geral para o particular (hierarquia deliberativa) e do particular para o geral (lógica distribuída, emergente).

O metasistema proposto foi organizado nos seguintes níveis: Sistema Geral, Sistema Específico e Caso Prático. A dinâmica do metasistema é multicausal, indo do geral para o particular e do particular para o geral. Em cada um destes níveis estão definidas variáveis e restrições. Partindo do geral para o particular, as variáveis vão sendo progressivamente menos abstratas e possuem mais restrições: algumas passam de variáveis livres para variáveis dependentes. No entanto, as variáveis do caso prático também podem influenciar o Sistema Específico e o Sistema Geral. Assim, o metasistema possui uma natureza hierárquica (do todo para a parte) e emergente (da parte para o todo). Enquanto a natureza hierárquica está mais associada a decisões deliberativas, a natureza emergente está mais associada a comportamentos distribuídos de autorregulação. A natureza deliberativa e distribuída do sistema será mais explícita quando forem descritas (para além das variáveis e dos processos de adaptação do metasistema), a adaptação estática e adaptação dinâmica. A palavra-chave na definição deste metasistema é retorno de informação, o que equivale, nos sistemas vivos, ao feedback.

\section{Caso prático, sistema Tetra Script}

No sistema responsivo Tetra Script é proposta uma membrana definida por componentes móveis que se abrem ou fecham para controlar a luz solar de acordo com fatores externos (localização geográfica, ano, mês, dia, hora, tipo céu) e internos (requisitos funcionais de iluminância e contraste visual, tipos de luz e padrões luz-sombra). Utiliza o adjetivo 'responsivo' por ser pensado para responder em tempo-real. Responsivo é um adjetivo que deriva do latim «respons?vu» que designa aquele que responde de forma rápida e adequada à situação. O processo de desenvolvimento está documentado numa tese de Doutoramento. $O$ objetivo deste artigo é retirar conclusões que permitam aplicar este método no desenvolvimento de outros sistemas responsivos.

\subsection{Variáveis, domínios e restrições}

Será explicada a organização em níveis, variáveis, domínio, restrições recorrendo ao exemplo do Sistema TetraScript No sistema geral as variáveis são mais abstratas, definindo qual o espaço de pesquisa e a focagem: desenvolver uma arquitetura com uma membrana de proteção que estabeleça uma relação holística entre o espaço interior e o espaço exterior, que seja personalizável através das tecnologias digitais. Pretende favorecer a utilização explícita de algoritmos, definindo parâmetros, descartando o contexto de implantação e topografia. Neste sistema geral poderiam ser definidos outros objetivos, como por exemplo a partição do espaço interior ou a aplicação deste princípio numa rede distribuída. Nesse sentido poderiam ser definidos diferentes graus de responsividade, sendo nesse caso necessário recorrer a métodos que assegurem uma gestão multicritério a nível superior.

No sistema específico o input inicial é uma superfície nurb contínua e o seu espaço topológico local, aos quais estão associados componentes planificáveis, que permitem definir aberturas móveis. Estas variáveis estão interligadas parametricamente através de um algoritmo. Quando o sistema específico é restringido a determinada caraterísticas é instanciado numa família de soluções (Fig. 4). Esta família numa linguagem de programação orientada a objetos seria designada como uma classe e teria os seus métodos.

O caso prático por sua vez pode ser instanciado numa construção específica. O pavilhão Tetra Script é uma instanciação do caso prático, fixados determinados valores. Para encontrar esses valores é utilizado um 
processo de adaptação estática. Na gestão dos objetivos do sistema é utilizada a adaptação dinâmica, que depois será pormenorizada. Fixando alguns dos valores como a localização geográfica e encontrada um tipo de superfície de acordo com a luz e o espaço disponível, podem ser definidas as caraterísticas e número de componentes.

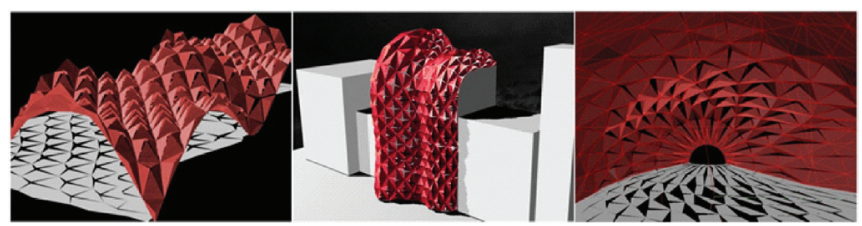

Figura 4: Sistema TetraScript: instâncias do mesmo caso específico.

No caso específico TetraScript a forma escolhida como componente base é uma pirâmide quadrangular, com as faces triangulares móveis e um material facilmente fabricável digitalmente. O pavilhão Tetra Script é uma instanciação de um sistema específico, restringindo as variáveis. E a procura da sua configuração resulta de um processo para encontrar a melhor relação entre as variáveis que satisfaça uma condição, definindo assim um espaço de procura de soluções progressivamente mais limitado. A maneira emergente de definir o sistema seria escolher primeiro um tipo de material e de componentes definindo depois a superfície, alterando eventualmente o sentido geral do sistema responsivo. Mas tal, como veremos, também pode ser conseguido uma solução emergente através de interação entre os componentes utilizando a adaptação dinâmica.

\begin{tabular}{|c|c|c|c|}
\hline \multirow{4}{*}{ Sistema Geral } & variável & domínio & restrição \\
\hline & $\begin{array}{c}\text { função } \\
\text { arquitetónica }\end{array}$ & $\begin{array}{l}\text { membrana } \\
\text { holística }\end{array}$ & $\begin{array}{c}\text { personalização } \\
\text { digital }\end{array}$ \\
\hline & algorítmico & $\begin{array}{c}\text { definição } \\
\text { paramétrica }\end{array}$ & $\begin{array}{l}\text { topografia/ } \\
\text { implantação }\end{array}$ \\
\hline & $\begin{array}{l}\text { sistema } \\
\text { holístico }\end{array}$ & $\begin{array}{c}\text { interação } \\
\text { micro/macro }\end{array}$ & $\begin{array}{c}\text { não } \\
\text { consideradas }\end{array}$ \\
\hline \multirow{3}{*}{$\begin{array}{c}\text { Sistema } \\
\text { Específico }\end{array}$} & superfície & $\begin{array}{c}\text { superfície } \\
\text { nurb continua }\end{array}$ & $\begin{array}{l}\text { espaço local } \\
\text { paramétrico }\end{array}$ \\
\hline & componentes & $\begin{array}{l}\text { definidos na } \\
\text { superfície }\end{array}$ & planificáveis \\
\hline & aberturas & $\begin{array}{l}\text { definidas nos } \\
\text { componentes }\end{array}$ & $\begin{array}{l}\text { aberturas } \\
\text { móveis }\end{array}$ \\
\hline \multirow{3}{*}{ Caso Prático } & $\begin{array}{l}\text { localização } \\
\text { específica }\end{array}$ & $\begin{array}{c}\text { Porto, } \\
\text { Portugal }\end{array}$ & $\begin{array}{l}41.158 \mathrm{~N} \\
-8.629 \mathrm{O}\end{array}$ \\
\hline & tipo superfície & sup. esferoide & $6 \times 4 \times 3 m$ \\
\hline & $\begin{array}{c}\text { componente } \\
\text { planificável }\end{array}$ & $\begin{array}{c}\text { pirâmide } \\
\text { quadrangular }\end{array}$ & $\begin{array}{l}\text { material } \\
\text { fabricável }\end{array}$ \\
\hline
\end{tabular}

Tabela 1: Definição por níveis do metasistema, no caso TetraScript.
Os níveis do metasistema são depois divididos em subníveis. No caso prático desenvolvido, depois de fixado um sistema específico foram definidos subníveis considerando grupos de variáveis definidos também em tabela que, por razões de espaço, aqui se sintetizam. Da interação entre cada um dos grupos de variáveis surge a resposta a cada caso específico. Os grupos de variáveis definidas foram: Variáveis externas ao objeto (tais como: local geográfico, data, tipo céu e respetivos domínios, restrições e dependências), as variáveis específicas da forma (tipo de superfície, dimensões, tipo de componentes e número), variáveis da forma do caso prático (área superfície, espaço paramétrico, domínio, número pirâmides, altura e valor de abertura) e as variáveis internas ou requisitos funcionais (Iluminância, conforto visual, conforto térmico e heurísticas espaciais). A dinâmica entre as variáveis é fundamental e é o que é feito escrevendo algoritmos.

\subsection{Adaptação estática e dinâmica}

O problema da adaptação do pavilhão ao contexto é uma questão de manipulação da sua forma relativamente a condições internas e externas, no curto e no longo prazo. São propostos dois processos de adaptação: a adaptação estática e a adaptação dinâmica. A adaptação estática controla a geração da geometria, até serem atribuídos valores aos parâmetros das variáveis e o pavilhão ser manufaturado e construído. A adaptação dinâmica controla a relação entre as partes após a implementação do sistema. Estes processos encontram paralelo nos sistemas vivos, na adaptação através da seleção natural e na adaptação comportamental, sendo apresentados alguns exemplos em publicações anteriores do autor.

Na adaptação estática vão sendo estabelecidas relações entre as variáveis, que vão sendo progressivamente discretizadas em valores. Como vimos no nível do sistema geral há objetivos que passam pela utilização dos processos digitais. Assim, foram utilizados num processo assíncrono e multisequencial os processos digitais CAD-CAE-CAM-AUT: a geração e manipulação de superfícies utilizando algoritmos, a fabricação testando tipos de componentes e agrupamentos, a simulação avaliando os desempenhos ambientais das escolhas da geração e a automação dos componentes desenvolvendo estratégias de implementação. Foi proposto um diagrama que descreve a interação entre ferramentas e processos digitais que poderá ser consultado em publicações anteriores (Henriques et al, 2012). A procura foi feita utilizando preferencialmente algoritmos explícitos, mas poderão ser aperfeiçoados e automatizados os processos de procura de soluções.

A adaptação dinâmica controla a relação entre as partes para assegurar o desempenho pretendido do sistema. Após a construção do pavilhão TetraScript a variável da forma que pode ser controlada é o grau de abertura dos painéis das claraboias, que assim determina a configuração formal do pavilhão. Há uma infinidade de combinações possíveis de aberturas para controlar a quantidade e o tipo de luz. A adaptação dinâmica permite encontrar configurações de abertura que satisfaçam requisitos de luz, nomeadamente 
iluminância e contraste visual. Este artigo irá fazer a descrição do processo que também pode ser consultado numa publicação anterior (Henriques et al, 2012).

São utilizadas heurísticas para encontrar espaços de soluções com determinadas caraterísticas que satisfaçam os requisitos pretendidos. As heurísticas são racionais matemáticos utilizados para identificar boas soluções em pouco tempo, em vez de soluções ótimas que nem sempre existem e que demoram mais a ser identificadas. As heurísticas descrevem padrões de comportamento de conjunto baseandoseem regras simples etipificando possíveis soluções, reduzindo assim o espaço de pesquisa. A pesquisa de otimização poderia fornecer melhores soluções, mas seria necessário muito tempo para que os resultados convergissem uma vez que o espaço de pesquisa de todas as configurações possíveis é muito vasto para poder ser analisado. O método de pesquisa heurística proposto engloba propriedades qualitativas e quantitativas da luz natural. As 5 heurísticas propostas estão descritas graficamente na fig.5.

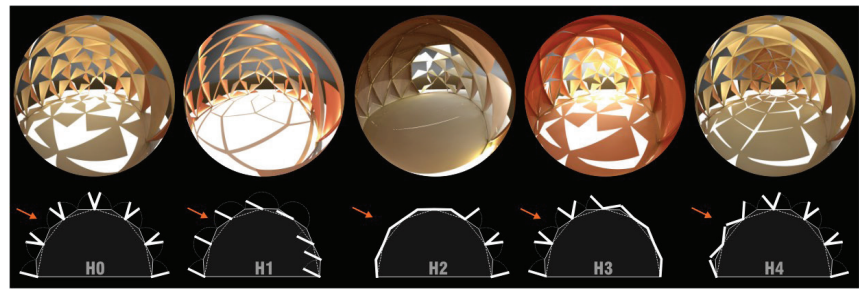

Figura 5: Visualização esquemática das heurísticas propostas. Em cima renders do interior de cada heurística, em baixo a representação esquemática. Da esquerda para a direita: HO abertura igual, H1 máxima luz direta, H2 luz difusa, H3 luz incidente, H4 híbrida baixa incidência + difusa. (Henriques et al, 2012).

No nível do caso prático há que considerar os grupos de variáveis referidos: as variáveis externas ao objeto, as variáveis específicas da forma, variáveis da forma do caso prático e variáveis internas ou requisitos funcionais. Para cada grupo há que descobrir as "sub-variáveis", os limites domínios e máximos. Ou seja, é necessário construir um sistema que se aproxima de um modelo matemático estabelecendo relações e limites. As heurísticas são definidas parametricamente sendo testadas as condições limite através da simulação para encontrar o espaço de soluções possíveis do sistema. Dito de forma simplificada, há que, entre outros fatores, fixar os requisitos de conforto visual e as condições de referência no exterior.

Foi utilizado o sistema paramétrico para encontrar configurações e um programa especializado (Radiance) para avaliar o desempenho. Foram realizadas simulações considerando todas as variáveis descritas anteriormente. Foram atribuídos valores e testado o desempenho do sistema através de programas de avaliação de iluminância e foi constituída uma base de dados de desempenho do TetraScript. Foi desenvolvido um método de interpolação para poder prever o comportamento lumínico noutras datas, horas e condições de céu diferentes das consideradas nos estudos realizados. Este método é utilizado para avaliar e escolher a configuração de abertura mais adequada ao tipo de condições interiores pretendidas. Foi desenvolvido um interface para controlar as condições internas do pavilhão, em tempo real, modificando a abertura dos painéis das claraboias.

\subsection{Interface controlo partilhado homem/máquina}

Algoritmo de controlo, feedback e adaptação. O usuário pode escolher a quais fatores atribuir maior relevância para personalizar o espaço, definindo a quantidade e qualidade da luz no interior do pavilhão através de um processo interativo. A personalização foi, precisamente, um dos objetivos da pesquisa realizada. Para escolher, o usuário é informado das várias possibilidades que o sistema oferece para se conseguir uma mesma quantidade de luz, com diferentes qualidades. Esta escolha pode ser efetuada em tempo real utilizando o interface proposto.

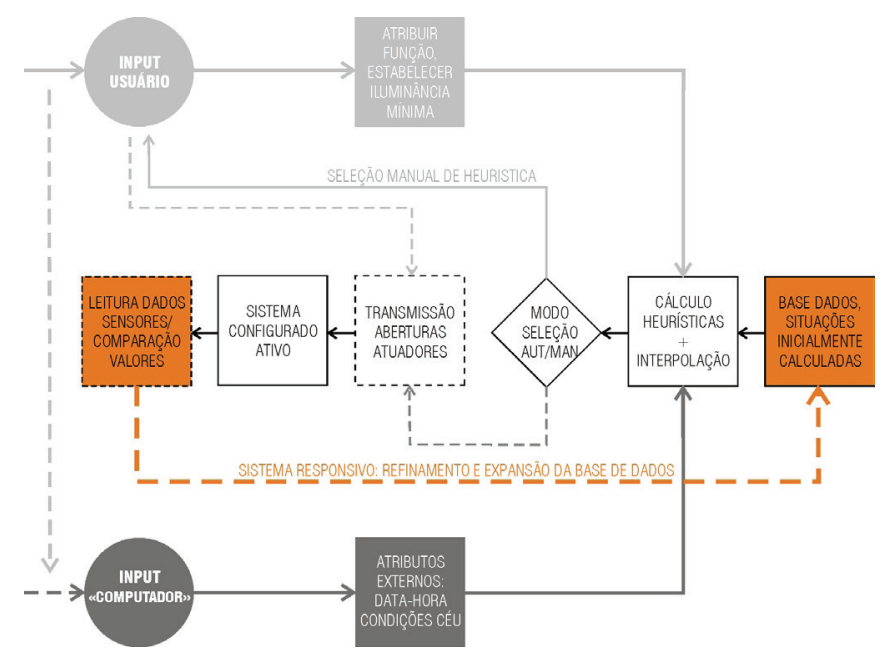

Figura 6: Sistema de controlo partilhado homem/máquina pavilhão TetraScript. (Henriques 2012).

Para controlar a abertura dos painéis das claraboias é proposto o esquema da Fig.6. Este sistema funciona com dois tipos de entradas: do usuário (manual) e do computador (automática). O usuário escolhe o mínimo de iluminância de acordo com a atividade que pretende. Esta informação é transmitida para o algoritmo, que calcula as várias configurações possíveis. $\mathrm{O}$ computador tem instruções para obter a informação da hora e local do computador que executa o programa e o conjunto desses inputs - iluminância necessária e condições da meteorológica e horárias - alimenta a busca heurística, que procura o valor através do método de interpolação. Em seguida, a escolha da solução mais adequada pode ser efetuado de maneira manual ou automática. No entanto, o aspeto mais significante deste esquema é o de adicionar um feedback loop, atualizando a base de dados com informações provindas de sensores de luz. Este 
processo permitirá que os valores esperados sejam refinados e otimizados para cada situação. Embora este processo ainda não esteja implementado, espera-se que com a sua utilização o sistema possa corrigir valores e, de certa forma, «aprenda com a experiência».

A natureza dual do metasistema deliberativo e indutivo cria espaços de possibilidades ("State spaces") que permitem encontrar soluções que vão para além das propriedades de cada parte, mas que resultam da sua interação. Assim, tanto o metasistema, nos seus diferentes níveis, como algoritmo de controlo procuram potenciar o imanente, o virtual, que só quando é explicitado se torna cognoscível, descobrindo possibilidades inteiramente novas.

\section{Resultados e discussão}

Este paper analisou e sintetizou contributos, procurando conceitos operativos para o desenvolvimento da arquitetura responsiva. Com base nestes contributos procurou incorporar conhecimentos de um caso de estudo anterior para propor um método para conceber sistemas responsivos. Tratando-se de uma tarefa complexa, pretende contribuir para um tema atualmente em discussão. $\mathrm{O}$ que ficou evidente nesta tentativa é a importância atual da computação no desenvolvimento e na implementação de sistemas.

Neste caso, a computação é utilizada para construir um modelo formal, que num certo sentido se aproximam mais de um modelo da física probabilística. Enquanto a física clássica apresenta um modelo em que cada objeto interage isoladamente, a física probabilística pelo contrário tende a valorizar a interação entre as partes em modelos de conjunto. A física probabilística considerar também várias causas em simultâneo, tendendo para uma multicausalidade que está ausente dos modelos determinísticos, de causalidade restrita (ou unicausais). O resultado da interação multicausal é previsível dentro de determinados parâmetros mas não é determinável a partida. Para potenciar estes sistemas a simples utilização do computador - ou computorização (como definida por Terzidis) não é suficiente, nem se pretende um apenas um sistema de representação da realidade. Ainda que o computador seja necessário como ferramenta, ganha relevância a computação, nomeadamente os métodos e ações utilizadas para conceber e operar sistemas.

\section{Agradecimentos}

Obrigado a todos que contribuíram para o desenvolvimento do TetraScript, a quem agradeço

\section{Bibliografia}

ACHTEN, Henry (2014). One and Many: An Agent Perspective on Interactive Architecture. Gerber, D. et al. (ed.), Proceedings 34th Annual Conference of the Association for Computer Aided Design in Architecture (ACADIA), Los Angeles 2014, 479-486.

ALEXANDER, Christoph (1991). "System Generating systems", em Architectural Design, December Issue No 7/6, John Willey \& Sons Ltd, London 1991, 90-1.

BERTALANFFY, Ludwig Von (1969) General System Theory: Foundations, Development, Applications, George Braziller, Nova Iorque.

DELANDA, Manuel (2011), Virtual Reality: in Computational Design Thinking, MENGES, Achim, AHLQUIST, Sean (edition). John Willey Sons Ltd, Chichester, Reino Unido, 142-8.

HENRIQUES, Gonçalo Castro (2012). TetraScript: A Responsive Pavilion, From Generative Design to Automation, International Journal of Architectural Computing, , Multi Science Publishing, Issue Volume 10, Number 1 / Março 2012, 87-104.

HENRIQUES, Gonçalo Castro, DUARTE, José Pinto e LEAL, Vítor, (2012) "Strategies to control daylight in a responsive skylights system", Automation in Construction, Volume 28, 91105.

HENRIQUES, Gonçalo Castro, Tese de Doutoramento (2013). Tetra Script: Sistema de aberturas responsivo para controlar a luz, de acordo com fatores externos e internos: Tese Doutoramento europeu apresentado na FAUL em Lisboa em 24 Junho 2013. Orientação José Duarte (FAUL) e Vítor Leal (FEUP). MAZRIA, Eduard (2003). It's the Architecture, Stupid! in magazine Solar Today. American Solar Energy Society, 2003, 48-51.

MENGES, Achim, AHLQUIST, Sean (2011). Computational Design Thinking, John Willey Sons Ltd, Chichester, Reino Unido.

NEGROPONTE, Nicholas (1975). Soft Architecture Machines, Cambridge, MA: MIT Press, 239.

PASK, Gordon (1969). The Architectural Relevance of Cybernetics", em Architectural Design, September issue No 7/6, John Wiley \& Sons, Londres.

STERK, Tristan d'Estree (2005). Building upon Negroponte: a hybridized model of control suitable for responsive architecture, in Journal Automation in Construction, v.14, no.2, 225-32.

STERK, Tristan d'Estree (2006). "Responsive Architecture: User-centered Interactions within the Hybridized Model of Control", In Proceedings of the Game Set and Match II: On Computer Games, Advanced Geometries and Digital Technologies, Netherlands, Episode Publishers, 494-501.

TERZIDIS, Kostas (2003), Algorithmic Architecture, Expressive Form: Spon Press, Taylor \& Francis Group. 